\title{
The Role of Environmental Factors and Mine Geometry in Thermal IR Mine Signatures
}

\author{
Brian A. Baertlein and İbrahim Kürşat Şendur \\ The Ohio State University, ElectroScience Laboratory \\ 1320 Kinnear Road, Columbus, OH 43212
}

\begin{abstract}
Thermal IR signatures of buried land mines are affected by various environmental conditions as well as the mine's composition, size and burial geometry. In this work we present quantitative relations for the effect of those factors on the signature's peak contrast and apparent diameter. We begin with a review of the relevant phenomena and the underlying physics. A three-dimensional simulation tool developed by the authors is used to simulate signatures for the case of a static water distribution. We discuss efforts to validate the model using experimental data collected at Fort A.P. Hill, VA. Using this simulation tool a variety of factors are considered, including soil water content, soil sand content, wind speed, mine diameter and mine burial depth.
\end{abstract}

Keywords: IR, thermal, mines, heat flow, modeling, simulation

\section{INTRODUCTION}

Anecdotal evidence and a growing body of experimental measurements suggest that buried mines are more detectable under certain environmental conditions. An analysis of the physics that describe these signatures suggests that a number of environmental factors as well as the local meteorological history play important roles. In addition, mines vary in size and burial depth, and it is well known that these mine-related factors affect the surface temperature distribution.

In this work we employ numerical modeling to quantify the aforementioned relations. A limitation of the simulations shown here is that the moisture distribution is assumed to be static, i.e., we ignore the effect of water and vapor movement on heat transfer and we ignore surface cooling through evaporation. In addition, we have linearized the analysis to simplify the simulations. In spite of these limitations, we are able to consider a number of important variables and to quantify their effect. Insight drawn from this work was used to improve signal processing techniques for buried mine detection, as described in a companion paper. ${ }^{1}$

The work is organized in three major parts. In Section 2 we review the physical processes that produce thermal mine signatures, including the role of moisture transport. These processes are controlled by a number of fundamental parameters including the soil thermal conductivity and diffusivity, the surface convection coefficient, the air temperature, and the incident solar radiance. We review models for these fundamental parameters that involve readily measured quantities. In Section 3 we briefly review our simulation tool and the simplification implied by a static moisture distribution. Work toward validating that tool is also described. In Section 4 we present a parameter study involving key environmental variables. The effects of those variables on surface temperature distribution are examined through simulation. We report both the shape of the surface temperature distribution and the time-dependence of the peak signature. The influence of the mine's diameter and depth on the signature are also studied. Concluding remarks appear in Section 5.

\section{COUPLED THERMAL-MOISTURE MODELING}

Heat transport is the primary phenomenon governing thermal IR signatures, but soil moisture content is also thought to have a significant role. Because of their importance in agriculture and remote sensing, the topics of heat and moisture transport in soils have an extensive literature. Seminal works in this area are those of Phillip and de Vries ${ }^{2}$ and de $\operatorname{Vries}^{3}$ (hereafter, referred to as PdV) who jointly presented a set of coupled partial differential equations that describe the transport phenomena. Numerous reviews, applications and extensions of that work have appeared.

Corresponding author: B.A.B. (614) 292-0076 (voice), (614) 292-7297 (fax), baertlein.1@osu.edu 
Because of the difficulties and computational expense involved in dealing with the resulting coupled nonlinear partial differential equations, all of the extant works are restricted to one-dimensional geometries.

Recent work with the PdV model includes that of Liou and England, ${ }^{4}$ who simulated surface temperatures during a 60-day dry-down period for use in modeling surface radio-brightness. Camillo et al. ${ }^{5}$ used a modified PdV formulation to predict measured soil temperatures and compared them to measurements. Scanlon and Milly ${ }^{6}$ used a new formulation of the PdV model ${ }^{7}$ to simulate water and heat transport in desert soils. Thomas and Sansom, ${ }^{8}$ drawing on prior work by Thomas and King, ${ }^{9}$ described a 1-D finite element model for the coupled transport problem. The only work in this area directly related to mine detection is apparently that of Pregowski et al. ${ }^{10}$ who presented a combination of numerical modeling and experimental measurement to assess the influence of soil moisture on buried mine signatures.

\subsection{Fundamental Equations}

Our description of the physics begins with a discussion of the partial differential equations that describe both heat and moisture transport in soil.

\subsubsection{Moisture Transport}

Consider first the case of isothermal conditions (i.e., no temperature gradient exists), in which all of the moisture is in the liquid phase (i.e., no vapor is present). Let $\theta_{\ell}(\mathbf{r}, t)$ be the volume fraction of liquid $\left[\mathrm{m}^{3} / \mathrm{m}^{3}\right]$, and let $\rho_{\ell}\left[\mathrm{kg} / \mathrm{m}^{3}\right]$ be the density of liquid, a quantity assumed invariant with space and time. We write

$$
X_{m}(\mathbf{r}, t)=\rho_{\ell} \theta_{\ell}
$$

where $X_{m}$ is the total water mass in a unit volume of soil $\left[\mathrm{kg} / \mathrm{m}^{3}\right]$. Darcy's equation allows us to write

$$
\mathbf{q}_{m}=-\rho_{\ell} K \nabla(\psi+z)
$$

where $\mathbf{q}_{m}$ is the moisture flux vector $\left[\mathrm{kg} \mathrm{m}^{-2} \mathrm{~s}^{-1}\right], z[\mathrm{~m}]$ is elevation, $\psi$ is the matric potential $[\mathrm{m}]$ and $K[\mathrm{~m} / \mathrm{s}]$ is the hydraulic conductivity. We have $\psi<0$ for unsaturated soils and $\psi=0$ for saturated conditions. The law of continuity

$$
\frac{\partial}{\partial t} X_{m}=-\nabla \cdot \mathbf{q}_{m}
$$

leads to a form of the Richards equation

$$
\frac{\partial \theta}{\partial t}=\nabla \cdot[K \nabla(\psi+z)]
$$

The presence of water vapor and temperature gradients introduces additional terms into this equation. The effects of these terms become more important near the soil surface, where the temperature gradients are higher. Since thermal mine signatures are based on soil surface temperature, these effects are highly relevant. Phillip and de Vries ${ }^{2}$ and de Vries ${ }^{3}$ generalized the Richards equation to address thermal and vapor transport. Let $\theta_{v}$ be the volume fraction of air $\left[\mathrm{m}^{3} / \mathrm{m}^{3}\right]$ (assumed to be water vapor) in the soil, and let $\rho_{v}\left[\mathrm{~kg} / \mathrm{m}^{3}\right]$ be the density of vapor. We write

$$
X_{m}(\mathbf{r}, t)=\rho_{\ell} \theta_{\ell}+\rho_{v} \theta_{v}
$$

Let $S\left[\mathrm{~m}^{3} / \mathrm{m}^{3}\right]$ be the porosity of the soil (i.e., the fraction of a volume that can be occupied by liquid or air). We have

$$
S=\theta_{\ell}+\theta_{v}
$$

We can define a total water content $\theta$ as follows

$$
\theta(\mathbf{r}, t)=\frac{X_{m}(\mathbf{r}, t)}{\rho_{\ell}}=\theta_{\ell}+\frac{\rho_{v}}{\rho_{\ell}}\left(S-\theta_{\ell}\right)
$$

Phillip and de Vries modified equation (2) by adding a vapor flux term

$$
\mathbf{q}_{m} / \rho_{\ell}=-K \nabla(\psi+z)-D_{v} \nabla \rho_{v}(\theta, T) / \rho_{\ell}
$$


where $D_{v}\left[\mathrm{~m}^{2} / \mathrm{s}\right]$ is the isothermal water vapor diffusion coefficient and $\rho_{v}$ is the absolute humidity of air in the soil matrix. In the original PdV formulation the matric potential $\psi$ and $\rho_{v}$ are both taken to be functions of $\theta$ and $T$. Applying the chain rule of differential calculus to the $\nabla$ operator leads to an equation for $\mathbf{q}_{m} / \rho_{\ell}$ in terms of $\theta$ and $T$.

Noting deficiencies in the PdV formulation, most notably that it fails to address the bulk flow of the air phase, Milly ${ }^{7}$ developed an alternative formulation using $\psi$ instead of $\theta *^{*}$ Starting with $\rho_{v}=\rho_{v}(\psi, T)$ and applying the chain rule for the spatial derivative of $\rho_{v}$ one obtains

$$
\begin{aligned}
\mathbf{q}_{m} / \rho_{\ell} & =-\left(K+D_{\psi v}\right) \nabla \psi(\mathbf{r}, t)-D_{T v}^{\prime} \nabla T(\mathbf{r}, t)-K \mathbf{k} \\
& =-K_{\psi m} \nabla \psi-K_{T m} \nabla T-J_{m} \mathbf{k} \\
K_{\psi m} & =K+D_{\psi v} \\
K_{T m} & =D_{T v}^{\prime} \\
J_{m} & =K
\end{aligned}
$$

in which we have defined the following diffusion coefficients

$$
\begin{aligned}
& D_{\psi v}=\left.D_{v} \frac{\partial \rho_{v}}{\partial \psi}\right|_{T} \\
& D_{T v}^{\prime}=\left.D_{v} f \frac{\partial \rho_{v}}{\partial T}\right|_{\psi}
\end{aligned}
$$

In these expressions the factor $f$ has been introduced, following $\mathrm{PdV}$, to account for larger temperature gradients in local liquid "islands" and in the air phase. Applying the conservation law leads to an analog of the Richards equation

$$
\frac{1}{\rho_{\ell}} \frac{\partial X_{m}}{\partial t}=\frac{1}{\rho_{\ell}} \frac{\partial}{\partial t}\left(\rho_{\ell} \theta_{\ell}+\rho_{v} \theta_{v}\right)=\frac{\partial \psi}{\partial t} M_{\psi m}+\frac{\partial T}{\partial t} M_{T m}
$$

where

$$
\begin{aligned}
& M_{\psi m}=\left.\left(1-\left(\frac{\rho_{v}}{\rho_{\ell}}\right)\right) \frac{\partial \theta_{\ell}}{\partial \psi}\right|_{T}+\left.\frac{\left(S-\theta_{\ell}\right)}{\rho_{\ell}} \frac{\partial \rho_{v}}{\partial \psi}\right|_{T} \\
& M_{T m}=\left.\left(1-\left(\frac{\rho_{v}}{\rho_{\ell}}\right)\right) \frac{\partial \theta_{\ell}}{\partial T}\right|_{\psi}+\left.\frac{\left(S-\theta_{\ell}\right)}{\rho_{\ell}} \frac{\partial \rho_{v}}{\partial T}\right|_{\psi}
\end{aligned}
$$

Some of the partial derivatives appearing in Eq. (16) and (17) can be evaluated in closed form. When a local equilibrium exists between the soil liquid and vapor, the vapor density is given by

$$
\rho_{v}(\psi, T)=\rho_{0}(T) h_{r}(\psi, T)
$$

where $\rho_{0}$ is the saturation vapor density and $h_{r}$ is the relative humidity, given by

$$
h_{r}(\psi, T)=\exp (\psi g / R T)
$$

in which $g=9.81\left[\mathrm{~m} \mathrm{~s}^{-2}\right]$ is the acceleration due to gravity and $R=461.5\left[\mathrm{~J} \mathrm{~kg}^{-1} \mathrm{~K}^{-1}\right]$ is the ideal gas constant for water vapor. Milly ${ }^{7}$ presents arguments that such an equilibrium will exist in soil under all conditions with the possible exception of rapid liquid infiltration through coarse soil (e.g., heavy rain on gravel).

Collecting these results, the following equation is obtained for the moisture transfer equation

$$
\frac{\partial \psi}{\partial t} M_{\psi m}+\frac{\partial T}{\partial t} M_{T m}=\nabla \cdot\left[K_{\psi m} \nabla \psi+K_{T m} \nabla T+J_{m} \mathbf{k}\right]
$$

${ }^{*}$ The PdV formulation also fails to address soil moisture hysteresis. The formulation of Milly attempted to address this problem, but it was later found to be flawed. ${ }^{6}$ 


\subsubsection{Thermal Transport}

The heat content $X_{h}\left[\mathrm{~J} / \mathrm{m}^{3}\right]$ of a unit volume of soil can be expressed in several forms, most of which can be related to that derived by de Vries $^{3}$

$$
\begin{aligned}
X_{h}(\mathbf{r}, t) & =\left(T-T_{0}\right) C+L_{v_{0}} \rho_{v} \theta_{a}-\rho_{l} \int_{0}^{\theta_{\ell}} W d \theta \\
C & =C_{d}+c_{\ell} \rho_{\ell} \theta_{\ell}+c_{v} \rho_{v} \theta_{v}
\end{aligned}
$$

where $T(\mathbf{r}, t)[\mathrm{K}]$ is the local temperature, $T_{0}[\mathrm{~K}]$ is a reference temperature, $C\left[\mathrm{~J} \mathrm{~m}^{-3} \mathrm{~K}^{-1}\right]$ is the volumetric heat capacity of moist porous soil, $C_{d}\left[\mathrm{~J} \mathrm{~m}^{-3} \mathrm{~K}^{-1}\right]$ is the volumetric heat capacity of dry porous soil, $c_{\ell}$ is the specific heat of liquid water at constant pressure, $c_{v}$ is the specific heat of water vapor at constant pressure, $L_{v}$ is the latent heat of vaporization at $T_{0}$, and $W$ is the differential heat of wetting. Following Edlefsen and Anderson ${ }^{11}$ we have

$$
W=-g(\psi-T d \psi / d T)
$$

Enforcing the conservation of energy leads to

$$
\frac{\partial X_{h}}{\partial t}=C \frac{\partial T}{\partial t}+L_{0} \rho_{\ell} \frac{\partial \theta_{v}}{\partial t}+c_{p} \rho_{\ell}\left(T-T_{0}\right) \frac{\partial \theta_{\ell}}{\partial t}-\rho_{\ell} W \frac{\partial \theta_{\ell}}{\partial t}=-\nabla \cdot \mathbf{q}_{h}
$$

where $\mathbf{q}_{h}$ is the thermal flux $\left[\mathrm{J} \mathrm{m}^{-2} \mathrm{~s}^{-1}\right]$.

The thermal flux has components due to conduction, latent heat in the vapor phase, and sensible heat in the liquid phase. Milly ${ }^{7}$ writes the formulation of de Vries $^{3}$ in the form

$$
\mathbf{q}_{h}=-\lambda \nabla T-\rho_{\ell} L_{v_{0}} D_{\psi v} \nabla \psi+c_{\ell}\left(T-T_{0}\right) \mathbf{q}_{m}
$$

where $\lambda\left[\mathrm{W} \mathrm{m}^{-1} \mathrm{~K}^{-1}\right]$ is the thermal conductivity of the porous, moist soil. He then adds to this flux the advection of the heat of wetting due to pressure head gradients, as suggested by Groevenelt and Kay ${ }^{12}$ to find

$$
\mathbf{q}_{h}=-K_{T h} \nabla T-K_{\psi h} \nabla \psi-J_{h} \mathbf{k}
$$

where

$$
\begin{aligned}
K_{T h} & =\lambda+c_{\ell}\left(T-T_{0}\right) \rho_{\ell} D_{T v}^{\prime} \\
K_{\psi h} & =-\rho_{\ell}\left(L_{v_{0}} D_{\psi v}+g T D_{T a}\right)+c_{\ell}\left(T-T_{0}\right) \rho_{\ell}\left(K+D_{\psi v}\right) \\
J_{h} & =c_{\ell}\left(T-T_{0}\right) \rho_{\ell} K
\end{aligned}
$$

In this result $D_{T a}$ is the diffusion coefficient for adsorbed water under the influence of a temperature gradient. Using $\theta_{v}=\theta_{v}(\psi, T)$ and $\rho_{v}=\rho_{v}(\psi, T)$ can derive expressions for the LHS of Eq. (24). Omitting the details, and combining the result with Eq. (26) we obtain for the heat transfer equation

$$
\frac{\partial}{\partial t} X_{h}(\mathbf{r}, t)=M_{T h} \frac{\partial T}{\partial t}+M_{\psi h} \frac{\partial \psi}{\partial t}=-\nabla \cdot \mathbf{q}_{h}=\nabla \cdot\left[K_{T h} \nabla T+K_{\psi h} \nabla \psi+J_{h} \mathbf{k}\right]
$$

in which

$$
\begin{aligned}
M_{T h} & =\left[C+H_{1} \frac{\partial \rho_{v}}{\partial T}+H_{2} \frac{\partial \theta_{\ell}}{\partial T}\right] \\
M_{\psi h} & =\left[H_{1} \frac{\partial \rho_{v}}{\partial \psi}+H_{2} \frac{\partial \theta_{\ell}}{\partial \psi}\right] \\
H_{1} & =\theta_{v}\left[L_{0}+c_{p}\left(T-T_{0}\right)\right] \\
H_{2} & =\left(c_{\ell} \rho_{\ell}-c_{p} \rho_{v}\right)\left(T-T_{0}\right)-\left(\rho_{\ell} W+\rho_{v} L_{0}\right)
\end{aligned}
$$




\subsection{Surface Boundary Condition}

The boundary condition for moisture transport at the air-soil interface must address both evaporation and precipitation. We have ${ }^{13}$

$$
\left.\frac{\mathbf{q}_{m} \cdot \mathbf{k}}{\rho_{\ell}}\right|_{z=0}=E-P
$$

where $E$ is the evaporation rate and $P$ is the precipitation. The evaporation rate is determined from the aerodynamic diffusion relation

$$
E=\frac{k^{2} U_{a} \phi}{\rho_{\ell}\left[\ln \left(z_{a} / z_{0}\right)\right]^{2}}\left(\left.\rho_{v}\right|_{z=0}-\rho_{v a}\right)
$$

where $k$ is von Karman's constant $(=0.4), z_{0}$ is the surface roughness length, $U_{a}$ is the wind speed measured at a reference height $z_{a}$, and $\rho_{v a}$ is the absolute vapor density measured at the same height.

The boundary condition for thermal transport comprises an energy balance. Approaches to this problem have been described by Camillo et al., ${ }^{5}$ Liou and England, ${ }^{14}$ and Milly, ${ }^{13}$ all of which are similar to

$$
\left.\mathbf{q}_{h} \cdot \mathbf{k}\right|_{z=0}=-(1-A) I_{s u n}-\epsilon I_{a t m}+\epsilon \sigma[T(z=0, t)]^{4}+\rho_{\ell}\left[L+c_{\ell}\left(T(z=0, t)-T_{0}\right)\right] E-\rho_{\ell} c_{\ell}\left(T_{a}-T_{0}\right) P+H
$$

where $A$ is the surface albedo at solar wavelengths, $I_{s}$ is the incoming solar radiation $\left[\mathrm{W} / \mathrm{m}^{2}\right], \epsilon$ is the soil emissivity at thermal wavelengths, $I_{a t m}\left[\mathrm{~W} / \mathrm{m}^{2}\right]$ is the longwave emission from the atmosphere to the earth, $\sigma$ is the StefanBoltzman constant, and $H$ is the convective exchange of sensible heat with the atmosphere. The heat absorbed by the soil is given by

$$
\left.\mathbf{q}_{h} \cdot \mathbf{k}\right|_{z=0}=-\left.\lambda\right|_{z=0} \frac{\partial T(z=0, t)}{\partial z}
$$

The convective losses $H$ are given by

$$
H=h_{c}\left[T(0, t)-T_{a}(t)\right]
$$

where

$$
h_{c}=\frac{C_{a} k^{2} U_{a} \phi}{\left[\ln \left(z_{a} / z_{0}\right)\right]^{2}}
$$

is the heat transfer coefficient for convection. In this expression $C_{a}$ is the volumetric heat capacity of the air at constant pressure, $T_{a}(t)$ is the temperature of the atmosphere at the surface, and $\phi$ is a stability factor related to the Richardson number. ${ }^{13}$ It is assumed that meteorological measurements of $U_{a}, I_{a t m}$, and $T_{a}$ are available. One can derive a similar form for the radiation terms. We have

$$
-\epsilon I_{a t m}+2 \epsilon \sigma\left[T(0, t)^{4}\right]=\epsilon \sigma\left[T_{a}(t)^{4}-T(0, t)^{4}\right]=h_{R}(T)\left[T_{a}(t)-T(0, t)\right]
$$

where

$$
h_{R}(T)=\epsilon \sigma\left[T_{a}(t)^{2}+T(0, t)^{2}\right]\left[T_{a}(t)+T(0, t)\right]
$$

is a temperature-dependent heat transfer coefficient for radiation losses.

\subsection{Parameter Models}

The foregoing relations involve numerous parameters, many of which depend on the variables $(\psi, T)$. This dependence leads to nonlinearities in the model. Unlike the transport of heat and moisture, which are based on well-known physical laws, parameter models are often based on measurements or empirical data.

Although the moisture model development is somewhat more straightforward that the thermal transport equations, its parameters are much more complex. Since our primary interest in this work lies with thermal transport, we briefly summarize some useful models for those parameters. In papers published during the $1970 \mathrm{~s}$, Mualem ${ }^{15,16}$ has provided what is perhaps the most widely used model for the volume fraction of water $\theta(\psi, T)$ and for the hydraulic conductivity $K$. Some useful approximate forms are also described by Milly. ${ }^{13}$ Expressions for the diffusion coefficients are also required and are described in the original PdV formulation. Drawing on prior work by de Vries, ${ }^{3}$ Milly ${ }^{13}$ presents expressions for $D_{\psi v}$ and for $D_{T v}^{\prime}$. The correction factor $f$ is provided by deVries. ${ }^{3}$ A model for the saturated water vapor density has been presented by Kimball et al. ${ }^{17}$ 
Many of the thermal parameters are derived from mixture models. Following de Vries, ${ }^{18}$ we write for the volumetric heat capacity $C$

$$
C=C_{\ell} \theta_{\ell}+C_{s}(1-S)+C_{v} \theta_{v}
$$

where $C_{u}$ is the heat capacity of component $u$. Some typical values for these constants have been tabulated. ${ }^{18}$

The volumetric thermal conductivity $\lambda$ is estimated from a similar mixture model ${ }^{18}$

$$
\lambda=\frac{\lambda_{\ell} \theta_{\ell}+\lambda_{s}(1-S) k_{s}+\left(\lambda_{a}+\lambda_{v a p}\right) \theta_{v} k_{a}}{\theta_{\ell}+(1-S) k_{s}+\theta_{v} k_{a}}
$$

where $k_{u}$ is the ratio of the space average of $\nabla T$ for particles of type $u$ to $\nabla T$ for water. Values of $k_{u}$ for spherical and cylindrical particles have been given. ${ }^{18}$ For spheres we have

$$
k_{u}=\frac{2}{3}\left[1+\left(\frac{\lambda_{u}}{\lambda_{w}}-1\right) g_{i}\right]^{-1}+\frac{1}{3}\left[1+\left(\frac{\lambda_{u}}{\lambda_{w}}-1\right)\left(1-2 g_{u}\right)\right]^{-1}
$$

in which the $g_{u}$ are shape factors for the constituents of soil. The value of $g_{u}$ has been tabulated for some solid soil constituents, and $g_{\ell}$ is not needed since it multiplies zero in $k_{\ell}$. The value of $g_{a}$ and values for $\lambda_{u}$ are given by Kimball et al. ${ }^{17}$

$$
\begin{aligned}
& \lambda_{v}=0.4186(0.0566+0.000153(T-273)) \\
& \lambda_{\ell}=0.4186\left(1.32+5.59 \times 10^{-3}\left((T-273)-2.63 \times 10^{-5}(T-273)^{2}\right)\right.
\end{aligned}
$$

Those authors also present an appraisal of methods for calculating $k_{a}$, a parameter with no physical meaning. It was proposed by De Vries that the thermal conductivity of air in the pore space be calculated as a mixture of air and water vapor vapor

$$
\lambda_{a v}=\lambda_{a}+\lambda_{v}
$$

The vapor conductivity is given by ${ }^{3}$

$$
\lambda_{v}=h_{r} L D_{a t m} \nu \frac{d \rho_{0}}{d T}
$$

where $L[\mathrm{~J} / \mathrm{kg}]$ is the latent heat of vaporization for water, ${ }^{17}$

$$
L=4186(595.9-0.548(T-273))
$$

$D_{a t m}$ is the molecular diffusivity of water vapor in the air ${ }^{17}$

$$
D_{\text {atm }}=0.229 \times 10^{-4}(T / 273)^{1.75}
$$

and $\nu$ is a mass flow factor that accounts for the mass movement of the soil air due to the unequal diffusion rate of air and water vapor molecules

$$
\nu=\frac{P}{P-p}
$$

In this expression $P$ is the barometric pressure and $p$ is the partial pressure of water vapor, given by

$$
p=h \rho_{0} R T / M_{w}
$$

in which $R$ is the gas constant (8314.3 [J kg-mole $\left.\left.{ }^{-1} \mathrm{~K}^{-1}\right]\right)$ and $M_{w}$ is the molecular weight of water $(18.016 \mathrm{~kg} / \mathrm{kg}$ mole). A model for the saturated water vapor density $\rho_{0}$ has been presented by ${ }^{17}$

$$
\rho_{0}(T)=10^{-3} \exp (19.819-4975.9 / T)
$$

The boundary conditions required additional parameters. Idso et al. ${ }^{19}$ note that soil albedo is a function of water content of the form

$$
A= \begin{cases}A_{d}+\left(A_{w}-A_{d}\right)\left(2 \theta_{\ell} / S\right) & 2 \theta_{\ell} / S<1 \\ A_{w} & 2 \theta_{\ell} / S<1\end{cases}
$$

and they present values for the constants. Values of the emissivity at IR wavelengths have been presented by Salisbury and D'Aria. ${ }^{20,21}$ The solar radiance $I_{s}$ and the attenuation of that radiance by the atmosphere can be computed using MODTRAN4, ${ }^{22}$ but a convenient closed form approximation is also available. ${ }^{23}$ 


\section{THERMAL MODELING FOR STATIC MOISTURE DISTRIBUTIONS}

\subsection{Prior Work}

The authors have previously described a combined thermal-radiometric model for the IR signature of a buried land mine. ${ }^{24}$ Three-dimensional thermal and radiometric models were developed to study the passive IR signature of a mine buried under a rough soil surface. That work is based on a linearized version of the model equations described in Section 2, and is further restricted to the case of a static, uniform water distribution. Evaporation and pressure gradients are ignored, but we retain the dependence of the parameters on the environmental factors, including $\theta_{\ell}$. In this case, the classical heat transfer equation is obtained

$$
\frac{\partial T(\mathbf{r}, t)}{\partial T}=\kappa(\mathbf{r}) \nabla^{2} T(\mathbf{r}, t)
$$

in which $\kappa=\lambda / C$ is the diffusivity, a coefficient that can be determined from environmental conditions. The solution of Eq. (56) also involves boundary conditions at the soil surface, which are given by the following linearized version of Eq. (37)

$$
-\left.\lambda\right|_{z=0} \frac{\partial T(z=0, t)}{\partial z} \approx-(1-A) I_{\text {sun }}-h_{R}\left[T_{a}(t)-T(z=0, t)\right]+h_{c}\left[T(z=0, t)-T_{a}(t)\right]
$$

We see that the boundary condition involves additional parameters including $\lambda$, the solar insolation $I_{\text {sun }}$, the convection heat transfer coefficient $h_{c}$, the radiative heat transfer coefficient $h_{R}$, and the local air temperature $T_{a}(t)$.

The numerical solution of Eq. (56) is based on the finite element method. The mine and soil are approximated by pentahedral elements, and $T$ is described by linear interpolation functions over those elements. The Crank-Nicholson algorithm is used for time-stepping the simulation. The behavior over a periodic diurnal cycle is studied by running the simulation to steady state. Finally, the finite element solution for the surface temperature is combined with reflected radiometric components to predict the signatures seen by an IR camera.

\subsection{Model Validation}

Recently, two efforts were undertaken to validate the model. In the first, we confirmed the validity of several approximations in the FEM formulation by developing an alternative solution, based on an integral equation formulation. Of particular interest were effects related to spatial discretization and boundary conditions, which are obviated in an integral equation formulation. The results of that study are documented separately, ${ }^{25}$ where it is shown that the FEM and integral equation code are in good agreement. Since the FEM formulation and the integral formulation have only the heat transfer equation in common, this agreement increases our confidence that both codes are correct.

We have also undertaken a comparison of the model with experimental data. Work to date has used 8-12 $\mu \mathrm{m}$ calibrated images provided by TRW. Those data comprise images of AT mines at times near 10:00A and 12:00 noon. The collections were done at the Fort. A.P. Hill, Site 71A mine lanes \#13 (dirt) and \#14 (gravel). Collateral information provided with the imagery included soil temperature at multiple depths $(0.5 ", 2$ ", 4 " and 8"), wind speed and direction, soil moisture content (one depth), air temperature, barometric pressure, and downwelling and upwelling radiance in the $0.3-3 \mu \mathrm{m}$ and $3-50 \mu \mathrm{m}$ bands.

To predict the measured data, it was necessary to make certain assumptions about unknown environmental parameters. Specifically, we assumed values for soil thermal conductivity $=1.8[\mathrm{~W} / \mathrm{mK}]$ and diffusivity $=10^{-6}\left[\mathrm{~m}^{2} / \mathrm{s}\right]$. For the mine under consideration here, an EM12 mine surrogate, we assumed a $30 \mathrm{~cm}$ diameter and $5.2 \mathrm{~cm}$ height. The mine's internal contents are not known, but some sources suggest that a combination of Styrofoam pellets and carnauba wax was used, which we modeled as a good thermal insulator. The resulting FEM mesh was comprised of pentahedral elements with cell dimension of $1.27 \mathrm{~cm}$ height and $2.56 \mathrm{~cm}$ base. A surface emissivity of 0.98 was assumed. MODTRAN4 was used to estimate the incident radiance as a function of time, and it was found to replicate measured values to an accuracy of about $10 \%$. Other environmental parameters were derived from the available data and models.

The measured data appear in Figure 1(a) after low-pass filtering to reduce small-scale image clutter related to surface roughness and emissivity variations. The results of our modeling effort appear in Figure 1(b), the shape of which is seen to compare well with Figure 1(a). A one-dimensional cut through the data is shown in Figure 2, which helps to quantify the agreement. We see that the signature shape and temperature contrast are fairly well 
predicted, but the model prediction is approximately $1 \mathrm{~K}$ below the measured data and it shows approximately $1 \mathrm{~K}$ less contrast. There are several possible sources for this error including our use of periodic boundary conditions in time. Warmer conditions the previous night can produce an offset that is not easily accounted for without additional data.
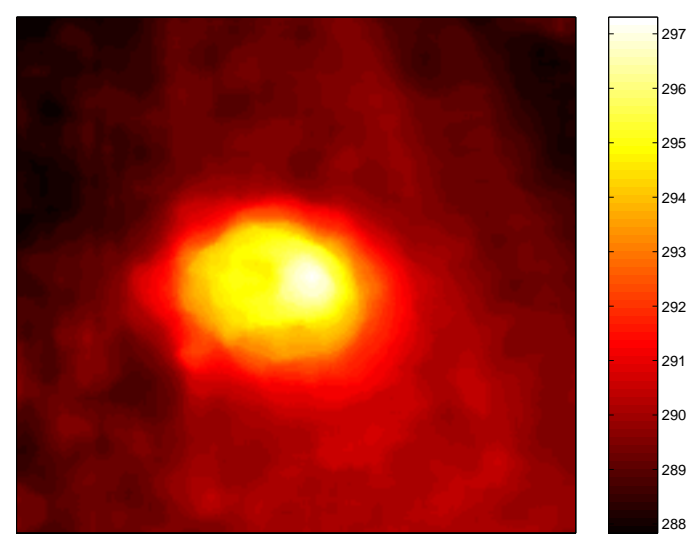

(a) Measured data after low-pass filtering to remove surface clutter

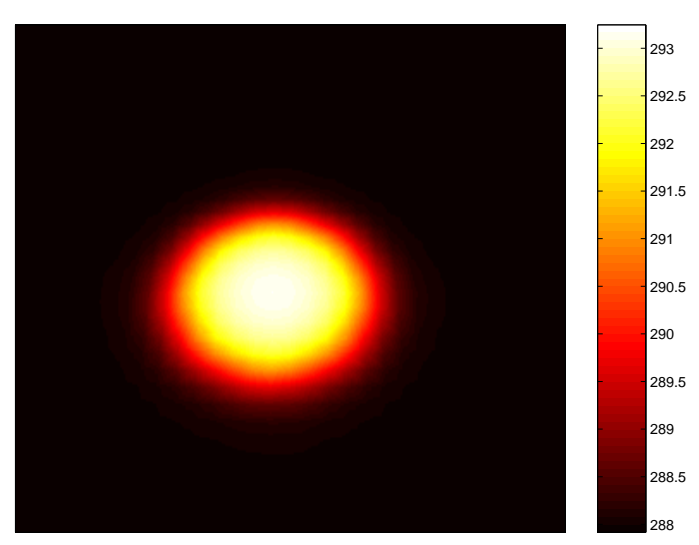

(b) Model prediction.

Figure 1. Measured and modeled results used in validation. The measurements were acquired at 12 noon on a dirt road over an EM12 mine surrogate.

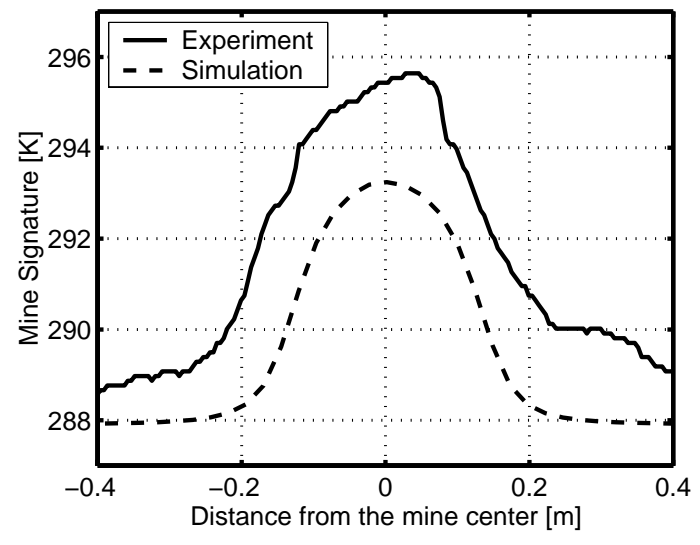

Figure 2. Comparison of model and experiment.

\section{PARAMETER STUDIES}

To quantify the effects of various environmental and mine-related parameters we have undertaken a numerical study of the model's sensitivity to these parameters. We used a baseline soil conductivity of $2.6 \mathrm{~W} \mathrm{~m}^{-1} \mathrm{~K}^{-1}$ and a diffusivity of $5 \times 10^{-7} \mathrm{~m}^{2} / \mathrm{s}$. For the mine parameters we used a conductivity of $0.23 \mathrm{~W} \mathrm{~m}^{-1} \mathrm{~K}^{-1}$ and a diffusivity of $9.3 \times 10^{-8}$ $\mathrm{m}^{2} / \mathrm{s}$. The dependence of the mine signature on some fundamental soil parameters is reviewed in Figures 3 and 4 . Some additional parameters are studied in a companion paper. ${ }^{1}$ In these results we have attempted to quantify the mine's surface signature using two parameters: the signature's surface temperature distribution and the maximum thermal contrast as a function of time. Additional studies reported in the companion paper suggest that, with the exception of very weak signatures that appear near the thermal cross-over time, the shape of the signature does not 
change substantially with time. For this reason, all plots of the spatial temperature distribution are normalized to unit magnitude as determined at a time of 1600 , when the signature attained its peak.

Thermal conductivity and diffusivity (or heat capacity) are the fundamental parameters which control thermal transport in soil. These parameters depend, in turn, on a number of environmental variables. We have selected soil water and quartz (sand) content as the parameters to be examined here. As noted above, water content has an important role in the physics. Quartz has a large thermal conductivity, which makes sand content a useful surrogate for soil thermal conductivity.

Figure 3 shows the shape and thermal contrast as a result of variations in soil sand content. The effect of varying water content is shown in Figure 4. It is evident that the spatial signature is essentially invariant to these parameters, while the contrast shows a weak effect. Variations in sand and water content have the effect of delaying the mines signature (or shifting its phase). This effect arises primarily as a result of changing the diffusivity of the soil.

Wind speed is another environmental variable that can affect the thermal signature via its effect on the convection coefficient. This parameter is considered in Figure 5. We see that the wind speed has little effect on the shape of the signature, but it has a more pronounced effect on the contrast. Unlike changes in soil parameters, however, it has no effect on the phase of the signature.

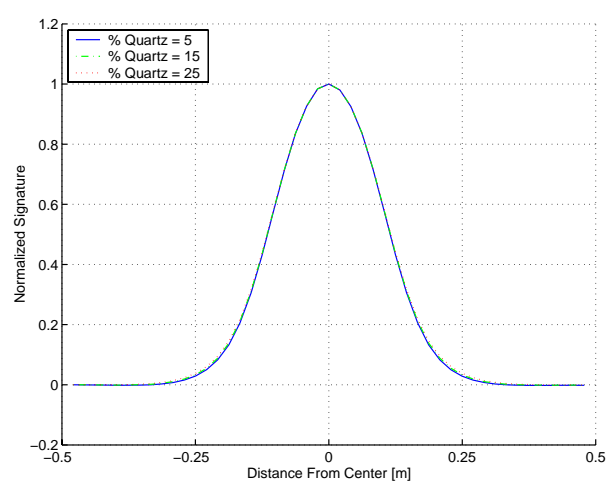

(a) Signature spatial dependence

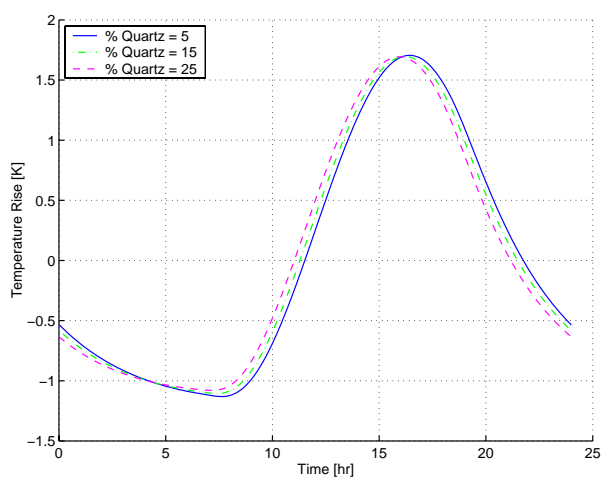

(b) Temporal variation in contrast over center of mine

Figure 3. Apparent size and temperature contrast produced by variations in soil sand content.

The effects of mine-related variables are reviewed in Figure 6 and 7. Variations in the mine diameter for a fixed depth are shown in Figure 6. It is found that the apparent signature diameter changes strongly with the mine diameter. A three-fold increase in the mine diameter from $10 \mathrm{~cm}$ diameter (AP mines) to $30 \mathrm{~cm}$ diameter (AT mines) produces less than a two-fold increase in the diameter of the signature at the surface. The temperature rise over the center of the mine, however, shows relatively little change over this range. Variations in burial depth (cf. Figure 7 appear to have little effect on the shape or size of the signature at the surface. The depth, however, has a significant effect on the temperature.

\section{SUMMARY AND CONCLUDING REMARKS}

We have reviewed the physics that relate environmental effects to thermal IR mine signatures. A model developed for the case of a static homogeneous moisture distribution was exercised to develop a quantitative relation among the signature shape, peak contrast, and various environmental and mine-related variables. We found that soil parameters have little effect on the shape of the signature, but they can make a more significant change in the amplitude and timing of the contrast peak. The wind speed can also affect the signature's peak contrast, but it has no effect on the signature shape or its temporal behavior. The depth of the mine has a significant effect on the amplitude of the contrast but, surprisingly, it has little influence on its shape. As expected, the mine diameter is tightly coupled to the shape of the surface temperature distribution. 


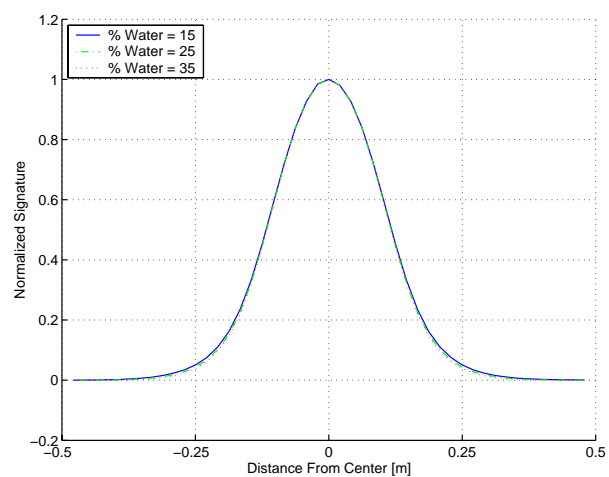

(a) Signature spatial dependence

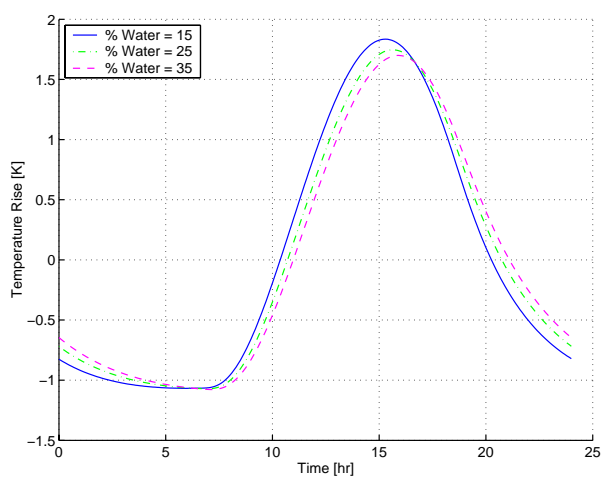

(b) Temporal variation in contrast over center of mine

Figure 4. Apparent size and temperature contrast produced by variations in soil water content.

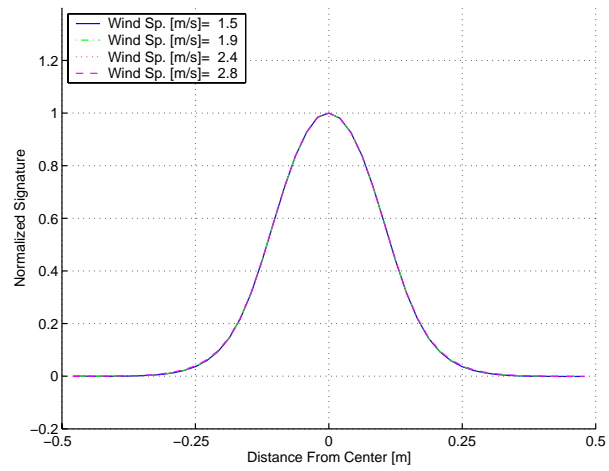

(a) Signature spatial dependence

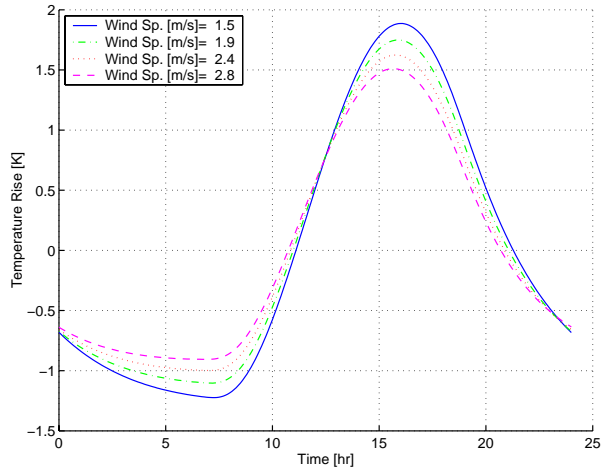

(b) Temporal variation of contrast over center of mine

Figure 5. Apparent size and temperature contrast produced by variations in wind speed.

\section{ACKNOWLEDGMENTS}

The authors would like to thank TRW for providing the experimental results, with special thanks to Ms. Sofia Monawer for her help in interpreting the data.

This project was supported by funds from Duke University under an award from the ARO (the OSD MURI program). The findings, opinions and recommendations expressed therein are those of the author and are not necessarily those of Duke University or the ARO.

\section{REFERENCES}

1. D.-H. Chen, I. K. Sendur, W.-J. Liao, and B. A. Baertlein, "Using physical models to improve thermal ir detection of buried mines," in Detection and Remediation Technologies for Mines and Minelike Targets VI, A. C. Dubey, J. F. Harvey, J. T. Broach, and R. E. Dugan, eds., SPIE 4394-26, 2001.

2. J. R. Phillip and D. A. de Vries, "Moisture movement in porous materials under temperature gradients," Trans. American Geophysical Union (Eos) 38(2), pp. 222-232, 1957. 


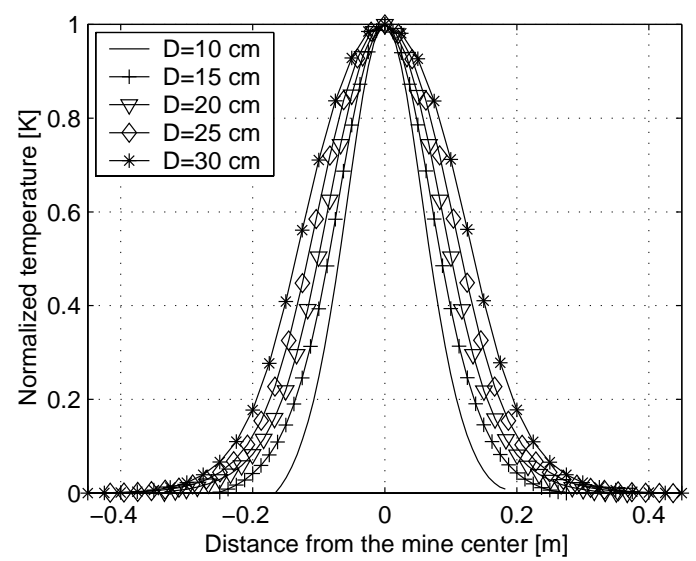

(a) Signature spatial dependence

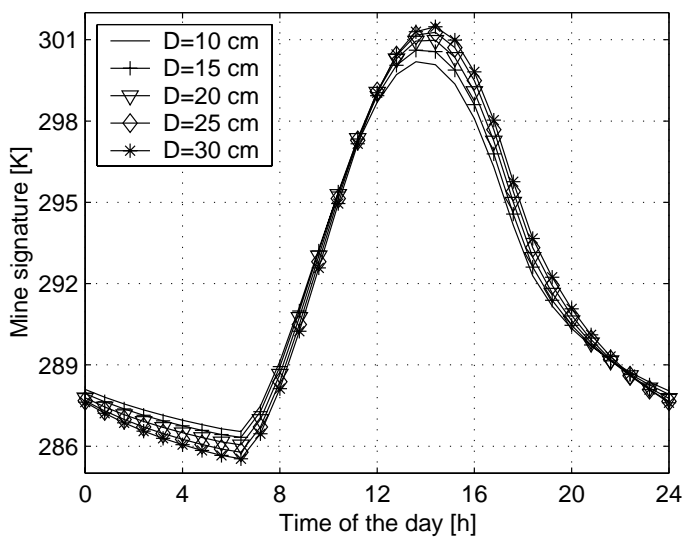

(b) Temporal variation over center of mine

Figure 6. The effect of mine diameter on apparent signature size and thermal time history.

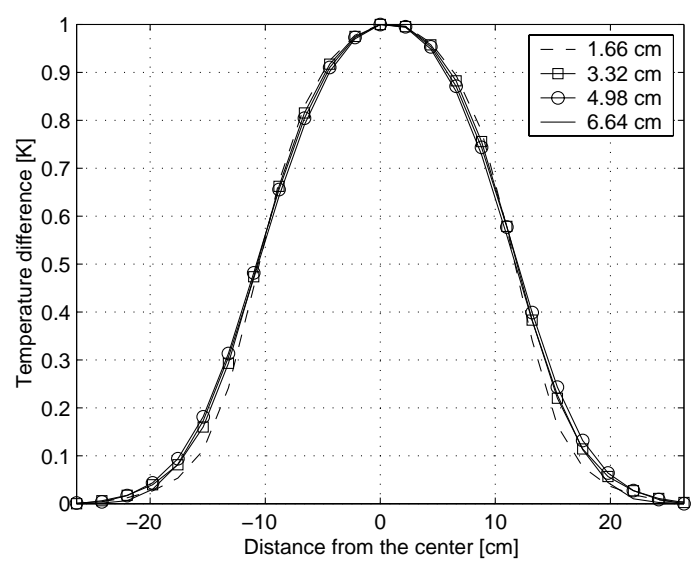

(a) Signature spatial dependence

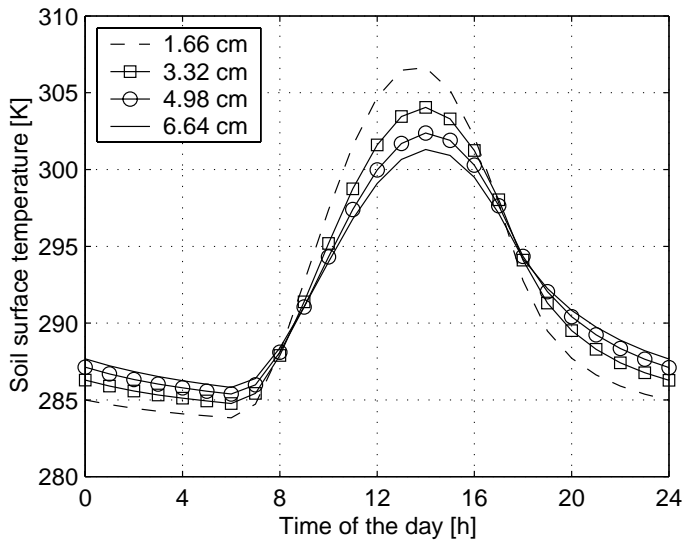

(b) Temporal variation over center of mine

Figure 7. The effect of burial depth on apparent signature size and thermal time history.

3. D. A. de Vries, "Simultaneous transfer of heat and moisture in porous media," Trans. American Geophysical Union (Eos) 39(5), pp. 909-916, 1958.

4. Y.-A. Liou and A. W. England, "A land surface process/radiobrightness model with coupled heat and moisture transport in soil," IEEE Transactions on Geoscience and Remote Sensing 36(1), pp. 273-286, 1998.

5. P. J. Camillo, R. J. Gurney, and T. J. Smugge, "A soil and atmospheric boundary layer model for evapotranspiration and soil moisture studies," Water Resources Research 19(2), pp. 371-380, 1983.

6. B. R. Scanlon and P. C. D. Milly, "Water and heat fluxes in desert soils 2. numerical simulations," Water Resources Research 30(3), pp. 721-733, 1994.

7. P. C. D. Milly, "Moisture and heat transport in hysteretic, inhomogeneous porous media: a matric head-based formulation and a numerical model," Water Resources Research 18(3), pp. 489-498, 1982.

8. H. R. Thomas and M. R. Sansom, "Fully coupled analysis of heat, moisture, and air transfer in unsaturated soils," J. Eng. Mech. 121, pp. 392-405, 1995. 
9. H. R. Thomas and S. D. King, "Coupled temperature/capilary potential variations in unsaturated soil," Journal of Engineering Mechanics 117(11), pp. 2475-2491, 1991.

10. P. Pregowski, W. Swiderski, R. T. Walczak, and K. Lamorski, "Buried mine and soil temperature prediction by numerical model," in Detection and Remediation Technologies for Mines and Minelike Targets V, A. C. Dubey, J. F. Harvey, T. J. Broach, and R. E. Dugan, eds., Proceedings of SPIE 4038, pp. 1392-1403, 2000.

11. N. E. Edlefsen and A. B. C. Anderson, "The thermodynamics of soil moisture," Hilgardia 16, pp. 31-299, 1943.

12. P. H. Groevenelt and B. D. Kay, "On the interaction of water and heat in frozen and unfrozen soils, 2. the liquid phase," Soil Sci. Soc. Am. Proc. 38(3), pp. 400-404, 1974.

13. P. C. D. Milly, "A simulation analysis of thermal effects on evaporation from soil," Water Resources Research 20(8), pp. 1087-1098, 1984.

14. Y.-A. Liou and A. W. England, "Annual temperature and radiobrightness signatures for bare soils," IEEE Transactions on Geoscience and Remote Sensing 34(4), pp. 981-998, 1996.

15. Y. Mualem, "Extension of the similarity hypothesis used for modeling the soil water characterstics," Water Resources Research 13(4), pp. 773-780, 1977.

16. Y. Mualem, "A conceptual model of hysteresis," Water Resources Research 10(3), pp. 514-520, 1974.

17. B. A. Kimball, R. D. Jackson, R. J. Reginato, F. S. Nakayama, and S. B. Idso, "Comparison of field-measured and calculated soil-heat fluxes," Soil Science Society of America Journal 40, pp. 18-25, 1976.

18. D. A. de Vries, "Heat transfer in soils," in Heat and mass transfer in the biosphere: Part 1. Transfer processes in the in the plant environment, D. A. deVries and N. H. Afgan, eds., ch. 1, pp. 5-28, Hemisphere Press, 1975.

19. S. B. Idso, R. D. Jackson, R. J. Reginato, B. A. Kimball, and F. S. Nakayama, "The dependence of bare soil albedo on soil water content," J. Applied Meteor. 14(1), pp. 109-113, 1975.

20. J. W. Salisbury and D. M. D'Aria, "Emissivity of terrestrial materials in the 8-14 $\mu \mathrm{m}$ atmospheric window," Remote Sensing Environment 42, pp. 83-106, 1992.

21. J. W. Salisbury and D. M. D'Aria, "Emissivity of terrestrial materials in the $3-5 \mu \mathrm{m}$ atmospheric window," Remote Sensing Environment 47, pp. 345-361, 1994.

22. A. Berk, L. S. Bernstein, and D. C. Robertson, "MODTRAN: a moderate resolution model for LOWTRAN7," Tech. Report GL-TR-89-0122, Air Force Geophysical Laboratory, Hanscom AFB, MA, October 211989.

23. A. W. England, "Radiobrightness of diurnally heated, freezing soil," IEEE Trans. Geosci. Remote Sensing 28(4), pp. 464-476, July 1990.

24. I. K. Sendur and B. A. Baertlein, "Simulation of thermal IR buried mine signatures," in Detection and Remediation Technologies for Mines and Minelike Targets V, A. C. Dubey, J. F. Harvey, T. J. Broach, and R. E. Dugan, eds., Proceedings of SPIE 4038, pp. 156-166, 2000.

25. I. K. Sendur and B. A. Baertlein, "Reference solution for thermal mine signature modeling," in Detection and Remediation Technologies for Mines and Minelike Targets VI, A. C. Dubey, J. F. Harvey, J. T. Broach, and R. E. Dugan, eds., SPIE 4394-24, 2001. 